\title{
Influência do local de origem do trauma nos índices de admissão de pacientes submetidos à laparotomia de emergência.
}

\section{Influence of trauma origin site on admission rates of patients submitted to emergency laparotomy.}

Luis Fernando Spagnuolo Brunello, AcCBC-PR'; Ana luísa Bettega, AcCBC-PR'; Phillipe Geraldo Teixeira de Abreu Reis, ACBC-PR'; Flavio Daniel Saavedra Tomasich, TCBC-PR'; I Inan Augusto Collaço, TCBC-PR'; Camilla Roginski Guetter, AcCBC-PR'; Thamyle Moda de Santana Rezende, AcCBC-PR'; Adonis Nasr, TCBC-PR'

\section{R E S U M O}

\begin{abstract}
Objetivo: avaliar a influência do local de ocorrência do trauma nos escores de trauma de pacientes submetidos à laparotomia de emergência. Métodos: estudo retrospectivo observacional analítico. Foram incluídos 212 pacientes submetidos à laparotomias exploratórias no período de janeiro de 2015 e dezembro de 2017. Informações sobre o local do acidente e dados vitais dos pacientes foram obtidas com base na coleta de dados por meio de prontuários eletrônicos e físicos. Foram analisados os índices de trauma de pacientes provenientes de Curitiba e Região Metropolitana e o local em que o paciente foi socorrido (estabelecimento físico ou via pública). Resultados: entre os 212 pacientes estudados, $184(86,7 \%)$ foram trazidos pelo Serviço de Atendimento Pré-Hospitalar provenientes da cidade Curitiba e 28 $(13,3 \%)$ provenientes de Região Metropolitana de Curitiba. Foram socorridos em estabelecimentos físicos 25 pacientes $(17,6 \%)$, enquanto $117(82,4 \%)$ foram socorridos em via pública. Observou-se maiores valores de ISS (Injurity Severity Score) dos pacientes procedentes da Região Metropolitana em relação aos procedentes de Curitiba $(29,78$ vs 22,46, $\mathrm{P}=0,009$ ), enquanto valores maiores do TRISS (Trauma Trauma and Injury Severity Score) foram observados em pacientes procedentes de Curitiba em relação aos da Região Metropolitana (90,62 vs 81,30; $P=0,015)$. Pacientes socorridos em via pública apresentaram menor valor de RTS (Revised Trauma Score) $(6,96$ vs 7,65; $\mathrm{P}=0,024)$ e TRISS $(86,42$ vs 97,21 ; $\mathrm{P}=0,012$ ). Conclusão: pacientes vítimas de trauma procedentes de locais mais distantes do atendimento no centro de referência apresentaram pior prognóstico à admissão e foi observado pior prognóstico em pacientes socorridos em via pública.
\end{abstract}

Descritores: Índices de Gravidade do Trauma. Traumatismos Abdominais. Laparotomia. Localização Geográfica de Risco. Epidemiologia Descritiva.

\section{INTRODUÇÃO}

A evolução dos sistemas de resgate e a possibilidade de iniciar intervenções na cena do trauma levaram a uma queda significativa nas taxas de mortalidade precoce pós-traumática, especialmente naquelas lesões que são diretamente associadas com o desenvolvimento de óbito precoce ${ }^{1}$. Estudos mostram que a densidade populacional é um preditor forte e independente de taxas de mortalidade municipal2,3. O tempo decorrido entre a injúria e o tratamento definitivo é importante e é razoável assumir que o tempo de transporte até o hospital parece ser um preditor independente de mortalidade. Alguns autores afirmam existir maior mortalidade em pacientes que moram em zonas rurais ${ }^{4}$. No entanto, estes estudos foram em maioria baseados em bancos de dados nacionais, não contendo dados anatômicos e fisiológicos das lesões, necessários para calcular os índices de trauma para avaliar a probabilidade de sobrevivência.

Uma das maneiras de avaliar a gravidade dos pacientes no trauma e poder predizer $\mathrm{O}$ prognóstico é por meio dos escores de trauma. Os principais exemplos de escores de trauma e que foram utilizados no artigo foram: Revised Trauma Score (RTS), Trauma and Injury Severity Score (TRISS) e Injurity Severity Score (ISS). Todos os escores citados são empregados na tentativa de estimar sobrevida e riscos de complicações, sendo utilizados na confecção de protocolos de atendimento ${ }^{5-7}$.

O conhecimento do perfil dos pacientes atendidos é de suma importância para a melhora do serviço prestado, facilitando o direcionamento de

1 - Hospital do Trabalhador, Serviço de Cirurgia Geral, Curitiba, PR, Brasil. 
políticas públicas. Dessa forma, este estudo objetivou avaliar a influência do local de ocorrência do trauma nos escores de trauma (ISS, RTS, TRISS) de pacientes submetidos à laparotomia exploradora de emergência em um hospital centro de referência de trauma do país.

\section{MÉTODOS}

Estudo transversal, analítico, observacional e com coleta de dados retrospectiva. Foram incluídos no estudo pacientes que foram submetidos à laparotomia exploratória no período de janeiro de 2015 a dezembro de 2017. O cenário do estudo foi a Sala de Emergência do Hospital do Trabalhador $(\mathrm{HT})$, que admite pacientes que chegam no hospital por procura referenciada (SIATE/SAMU). A coleta de dados foi realizada de forma retrospectiva a partir do prontuário eletrônico, sendo que os autores desenvolveram ficha de campo específica para este estudo contemplando dados sociodemográficos, registro de atendimento pré-hospitalar, dados clínicos, diagnóstico, tratamento (cirurgia), pós-operatório, sobrevida e índices de trauma. Foram excluídos do estudo os casos de pacientes com prontuário inacessível, falta de informações pertinentes para a realização da pesquisa, menores de 18 anos de idade, pacientes que não foram levados ao hospital por atendimento pré-hospitalar e pacientes que não foram submetidos à laparotomia exploradora.

Os dados coletados foram classificados e analisados de duas maneiras diferentes: a primeira correspondeu ao local de ocorrência do trauma (Curitiba ou Região Metropolitana) e a segunda correspondeu ao estabelecimento (físico ou via pública) no qual o paciente foi localizado e assistido pela primeira vez após o trauma. Entende-se por Região Metropolitana todos os municípios que, por processo de conurbação, tornaram-se próximos à capital paranaense. Os dados foram coletados retrospectivamente entre janeiro de 2017 e março de 2018, foram compilados utilizando formulário
Google Docs $®$ de maneira online e analisados por meio da plataforma online aberta Socscistatistics $₫$.

Características clínicas e demográficas com distribuição normal foram descritas utilizando média e desvio padrão. Dados epidemiológicos foram calculados com porcentagens estatísticas simples. As variáveis contínuas foram analisadas com o teste t de Student e as variáveis categóricas, por meio do teste qui-quadrado; sendo o nível de significância definido como 5\%. O presente estudo foi aprovado pelo Comitê de Ética do Hospital do Trabalhador, tendo o parecer no 1.802.091.

\section{RESULTADOS}

No período estudado, foram submetidos à laparotomia exploradora 413 pacientes, dos quais 201 foram excluídos do estudo. Dentre os 212 pacientes estudados, 184 (86,7\%) foram trazidos pelo Serviço de Atendimento PréHospitalar provenientes da cidade de Curitiba e 28 $(13,3 \%)$ provenientes de Região Metropolitana de Curitiba. Entre os pacientes estudados, houve predominância do sexo masculino (88,7\%) e média de idade de 28,7 anos (Tabela 1).

Em relação aos mecanismos de trauma, ferimento por arma de fogo (FAF) foi responsável por $111(52,3 \%)$ traumas, ferimento por arma branca (FAB), por 53 (25\%), acidentes automobilísticos, por $31(14,6 \%)$, quedas, por três $(1,4 \%)$, e outros mecanismos, por quatro $(1,8 \%)$ traumas. Não foi encontrado este dado no prontuário de dez $(4,7 \%)$ pacientes. Segundo à localidade de ocorrência do trauma, foram encontrados, em Curitiba, 88 (47,8\%) FAF, 50 (27,1\%) FAB, 29 (16,3\%) acidentes, três $(1,6 \%)$ quedas, três $(1,6 \%)$ traumas por outros mecanismos e dez $(5,4 \%)$ pacientes sem este dado registrado. Na Região Metropolitana foram encontrados 23 (82,1\%) FAF, três $(10,7 \%)$ $F A B$, um $(3,5 \%)$ acidente e um $(3,5 \%)$ trauma por outros mecanismos. 
Foram observados maiores valores de ISS dos pacientes procedentes da Região Metropolitana em relação aos procedentes de Curitiba $(P=0,009)$. Valores maiores do TRISS foram observados em pacientes procedentes de Curitiba em relação aos da Região Metropolitana $(P=0,0155)$. Não foi observada diferença estatisticamente significativa entre RTS das duas localidades ( $P=0,405)$ (Tabela 2).

Houve $14,6 \%$ de óbitos em pacientes provenientes de Curitiba e $25 \%$ de óbitos em pacientes procedentes da Região Metropolitana. Apesar da tendência à maior mortalidade na Região Metropolitana, não foi observada diferença significativa de óbitos entre Curitiba e Região
Metropolitana $(P=0,165)$. Por outro lado, o tempo de transporte do local de ocorrência do trauma até o hospital de referência foi maior na Região Metropolitana em relação à Curitiba $(P<0,001)$ (Tabela 3).

Em relação ao estabelecimento no qual o paciente foi assistido pela primeira vez após o trauma, houve predomínio de atendimentos em via pública (182 pacientes, 85,8\%), seguido por atendimento em Unidades Básicas de Saúde (UBS) ou Unidades de Pronto Atendimento (UPA) (18 pacientes, 8,4\%). Não houve óbitos documentados em pacientes atendidos em seus locais de trabalho ou domicílio. Pacientes socorridos em via pública apresentaram menor valor

Tabela 1. Dados epidemiológicos.

\begin{tabular}{lccc}
\hline & Amostra $(\mathrm{N}=212)$ & Curitiba $(\mathrm{N}=184)$ & Região Metropolitana $(\mathrm{N}=28)$ \\
\hline Sexo $(\%)$ & & & \\
Masculino & $188(88,7)$ & $162(88)$ & $26(92,8)$ \\
Feminino & $24(11,3)$ & $22(12)$ & $2(7,2)$ \\
Idade (média) & 28,7 & 29 & 26,6 \\
Mecanismo do trauma (\%) & & & $23(82,1)$ \\
FAF & $111(52,3)$ & $88(47,8)$ & $3(10,7)$ \\
FAB & $53(25)$ & $50(27,1)$ & $1(3,5)$ \\
AAuto & $31(14,6)$ & $30(16,3)$ & 0 \\
Quedas & $3(1,4)$ & $3(1,6)$ & $1(3,5)$ \\
Outros & $4(1,8)$ & $3(1,6)$ & 0 \\
Não informado & $10(4,7)$ & $10(5,4)$ & \\
\hline
\end{tabular}

$N=$ número da amostra; $D P=$ desvio padrão; $F A F=$ ferimento por arma de fogo; $F A B=$ ferimento por arma branca; AAuto $=$ acidentes automobilísticos.

Tabela 2. Índices de trauma do local de ocorrência do trauma.

\begin{tabular}{lccc}
\hline & Curitiba $(\mathrm{N}=184)$ & Região Metropolitana $(\mathrm{N}=28)$ & $\mathrm{P}(\mathrm{IC} 95 \%)$ \\
\hline ISS (DP) & $22,46( \pm 14,59)$ & $29,78( \pm 19,49)$ & $0,009 *$ \\
RTS (DP) & $7,143( \pm 1,61)$ & $7,121( \pm 1,25)$ & 0,405 \\
TRISS (DP) & $90,62( \pm 19,44)$ & $81,30( \pm 30,37)$ & $0,015^{*}$ \\
\hline
\end{tabular}

$N=$ número da amostra; $D P=$ desvio padrão. ${ }^{*}=$ resultado estatisticamente significativo.

Tabela 3. Taxa de óbitos e tempo de transporte do local de ocorrência do trauma.

\begin{tabular}{lccc}
\hline & Curitiba $(\mathrm{N}=184)$ & Região Metropolitana $(\mathrm{N}=28)$ & $\mathrm{P}(\mathrm{IC} 95 \%)$ \\
\hline Óbitos (\%) & $27(14,6)$ & $7(25)$ & 0,165 \\
Tempo de transporte em minutos (DP) & $37,97( \pm 20,15)$ & $112,0( \pm 90,06)$ & $<0,001^{*}$ \\
\hline
\end{tabular}

$N=$ número da amostra; $D P=$ desvio padrão; * = resultado estatisticamente significativo. 
de RTS $(P=0,034)$ e TRISS $(P=0,023)$ em relação aos socorridos em um estabelecimento físico. Não foi observada diferença estatisticamente significativa entre número de óbitos e ISS entre pacientes assistidos nessas cenas de trauma (Tabela 4).

\section{DISCUSSÃO}

Para este estudo, assim como previsto na literatura, foram utilizados os índices de trauma para avaliar a possibilidade de sobrevivência do paciente politraumatizado e também quantificar as suas modificações fisiológicas e lesões anatômicas. A amostra foi constituída por pacientes submetidos apenas à laparotomia exploradora, objetivando avaliação direta dos índices de trauma com maior fidelidade. Dois dos três índices avaliados (TRISS e RTS) mostraram uma associação positiva com o local de ocorrência do trauma em que foi encontrado ISS maior nos pacientes provenientes da Região Metropolitana, indicando estes estarem mais gravemente feridos, e maior TRISS nos pacientes provenientes da capital, representando maior chance de sobrevida para estes pacientes ${ }^{8}$.

Não foram incluídos neste estudo pacientes admitidos por procura direta, apenas aqueles por procura referenciada com atendimento pré-hospitalar. Desta maneira, o tempo de transporte foi calculado pela diferença dos horários registrados no Relatório de Atendimento do Socorrista (RAS) e o Boletim de Emergência (BE) emitido pelo hospital no momento da admissão. Embora houvesse diferenças entre os tempos de transporte até o hospital dos pacientes provenientes da Região Metropolitana em relação aos provenientes de Curitiba (112min. vs 37min., $\mathrm{P}<0,001)$, o número de óbitos entre essas localidades não apresentou diferença significativa $(P=0,165)$. Em estudo de coorte realizado com 19.167 pacientes $^{9}$, foi constatado que o tempo de transporte até o hospital de referência tem influência na sobrevida dos pacientes politraumatizados, no entanto, os autores do estudo encontraram essa associação com tempo de transporte menor do que 20 minutos. O presente estudo não mostrou diferenças entre a taxa de mortalidade para transporte com tempo superior a 20 minutos.

O aumento da espera para tratamento definitivo pode influenciar na piora neurológica da vítima, na instabilidade hemodinâmica e, consequentemente maior risco de PCR, dentre outras condições ${ }^{10}$. No entanto, apesar de os pacientes provenientes da Região Metropolitana apresentar quadro mais grave na chegada ao pronto atendimento (menor TRISS), este estudo não encontrou relação entre o local de ocorrência do trauma com mortalidade. Além disso, observouse um maior ISS nos pacientes provenientes de regiões metropolitanas, que indica pacientes mais gravemente feridos. Isto pode ser decorrente da maior taxa de criminalidade na região metropolitana.

Em relação ao estabelecimento no qual o paciente vítima de trauma foi socorrido, observou-se maior índice de lesões anatômicas

Tabela 4. Estabelecimento onde paciente foi socorrido.

\begin{tabular}{lcccc}
\hline & Óbitos $(\%)$ & ISS $(\%)$ & RTS $(\%)$ & TRISS $(\%)$ \\
\hline Via pública (N=117) & $23(19,6)$ & $26,06( \pm 18,06)$ & $6,96( \pm 1,72)$ & $86,42( \pm 24,32)$ \\
Estabelecimento físico (N=25) & 2 & $18,80( \pm 8,91)$ & $7,65( \pm 0,50)$ & $97,21( \pm 2,56)$ \\
UBS/UPA (N=18) & $2(11,1)$ & $18,88( \pm 9,21)$ & $7,61( \pm 0,58)$ & $97,08( \pm 2,52)$ \\
Local de trabalho (N=3) & 0 & $19,66( \pm 12,89)$ & $7,841( \pm 0)$ & $96,23( \pm 4,72)$ \\
Domicílio (N=4) & 0 & $18,0( \pm 7,14)$ & $7,693( \pm 0,33)$ & $98,26(0,80)$ \\
P (IC 95\%) & 0,164 & $0,024^{*}$ & $0,024^{*}$ & $0,012^{*}$ \\
\hline
\end{tabular}

$N=$ número da amostra; $D P=$ desvio padrão; * $=$ resultado estatisticamente significativo. 
(ISS) nos pacientes que foram atendidos em via pública quando comparados aos atendidos em algum tipo de estabelecimento físico $(P=0,024)$. Concomitantemente, pacientes atendidos em via pública apresentaram piores condições clínicas fisiológicas pelo índice RTS ( $P=0,024)$ e menor probabilidade de sobrevivência pelo índice TRISS $(P=0,012)$ quando comparados aos pacientes atendidos em estabelecimento físico. É provável que as condições ambientais e o tempo - muitas vezes incerto - do momento do trauma em pacientes socorridos em via pública são fatores que contribuem significativamente para pior prognóstico no momento do atendimento hospitalar.

Por outro lado, há poucos estudos que relacionam prognóstico e o local em que a vítima de trauma foi encontrada pela equipe de atendimento pré-hospitalar. Embora este estudo não tenha encontrado associação entre número de óbitos hospitalares em pacientes socorridos nos diferentes locais, espera-se que um estabelecimento físico, seja ele domiciliar ou Unidades de Saúde de atenção primária, seja mais favorável à estabilização clínica e encaminhamento rápido para o centro de referência em trauma mais próximo. Esta atuação precoce e eficiente no manejo do paciente pode não ser capaz de melhorar a sobrevida com base nos índices de trauma levantados, mas pode ser fundamental em manter as taxas de óbito semelhantes entre os dois locais. Portanto, são necessários novos estudos que avaliem a qualidade de atendimento hospitalar e pré-hospitalar para comprovar a semelhança proporcional de taxa de óbitos entre as duas localidades.

Este trabalho tem como limitação ser baseado em registros que não foram desenhados para o estudo, como o Relatório de Atendimento do Socorrista (RAS), o que compromete a qualidade dos dados em alguns casos. Também foi frequente a falta de dados para o preenchimento da ficha de coleta por potenciais fatores de confusão, uma vez que os dados foram registrados no passado. Perdas de seguimento do paciente também podem representar viés para estudos retrospectivos como este.

O modelo de estudo proposto mostrou que pacientes vítimas de trauma procedentes da região metropolitana apresentaram pior prognóstico com base nos índices de trauma calculados. Concomitantemente, também foi observado pior prognóstico em pacientes socorridos em via pública quando comparados com pacientes socorridos em um estabelecimento físico.

\section{A B S T R A C T}

Objective: to evaluate the influence of the site of trauma occurrence on the trauma scores of patients submitted to emergency laparotomy. Methods: this is a retrospective, observational, analytical study. We included 212 patients who underwent exploratory laparotomies in the period of January 2015 and December 2017. We obtained information about the accident site and vital data of the patients based on data collection through electronic and physical records. We analyzed the trauma indices of patients from Curitiba and its Metropolitan Region and the place where the patient was rescued (physical establishment or public road). Results: among the 212 patients studied, 184 (86.7\%) were brought by the Prehospital Care Service from the city of Curitiba, and 28 (13.3\%), from the Metropolitan Region of Curitiba. Twenty-five patients (17.6\%) were rescued in physical establishments, while 117 (82.4\%) were rescued on public roads. We observed higher values of Injurity Severity Scores (ISS) in patients coming from the Metropolitan Region than in those coming from Curitiba (29.78 vs 22.46, $P=0.009)$, but higher values of Trauma and Injury Severity Scores (TRISS) in patients from Curitiba than the ones from the Metropolitan Region (90.62 vs 81.30, $P=0.015$ ). Patients rescued in public roads presented lower Revised Trauma Scores (RTS) (6.96 vs 7.65, $P=0.024$ ) and TRISS (86.42 vs 97.21; $P=0.012)$. Conclusion: trauma victims from sites more distant from the referral center and rescued on public roads presented worse prognosis.

Keywords: Trauma Severity Indices. Abdominal Injuries. Laparotomy. Geographical Localization of Risk. Epidemiology Descriptive. 


\section{REFERÊNCIAS}

1. Regel G, Stalp M, Lehmann U, Seekamp A. Prehospital care, importance of early intervention on outcome. Acta Anaesthesiol Scand Suppl. 1997;110:71-6.

2. Kristiansen $T$, Lossius HM, Rehn M, Kristensen P, Gravseth HM, Røislien J, et al. Epidemiology of trauma: A population-based study of geographical risk factors for injury deaths in the working-age population of Norway. Injury. 2014;45(1):23-30.

3. Røislien J, Lossius HM, Kristiansen T. Does transport time help explain the high trauma mortality rates in rural areas? New and traditional predictors assessed by new and traditional statistical methods. Inj Prev. 2015;21(6):367-73.

4. Chen HY, Ivers RQ, Martiniuk AL, Boufous S, Senserrick $T$, Woodward M, et al. Socioeconomic status and risk of car crash injury, independent of place of residence and driving exposure: results from the DRIVE Study. J Epidemiol Community Heal [Internet]. 2010 Nov [cited 2018 May 5];64(11):998-1003. Available from: http:// jech.bmj.com/cgi/doi/10.1136/jech.2009.091496.

5. Baker SP, O'Neil B, Haddon W Jr, Long WB. The injury severity score: a method for describing patients with multiple injuries and evaluating emergency care. J Trauma [Internet]. 1974 Mar [cited 2018 May 5];14(3):187-96. Available from: http://content.wkhealth.com/ kbackndingpage\&an=00005373-197403000-00001

6. Alvarez BD, Razente DM, Lacerda DAM, Lother NS, Von Bahten LC, Stahlschmidt CM. Analysis of the Revised Trauma Score (RTS) in 200 victims of different trauma mechanisms. Rev Col Bras Cir [Internet]. 2016 Sep./Oct. [cited 2018 May 5];43(5):334-40. Available from: http://www.
scielo.br/scielo.php?script=sci_arttext\&pid=S010069912016000500334\&lng=en\&tlng=en

7. Orhon R, Eren SH, Karadayi S, Korkmaz I, Coskun $A$, Eren $M$, et al. Comparison of trauma scores for predicting mortality and morbidity on trauma patients. Ulus Travma Acil Cerrahi Derg. 2014;20(4):258-64.

8. Pereira Júnior GA, Scarpelini S, Basile-Filho A, Andrade Jl de. Índices de trauma. Medicina, Ribeirão Preto (Online).1999 Sep [cited 2018 May 5];32(3):237-50. Available from: http://revista.fmrp. usp.br/1999/vol32n3/indices_trauma.pdf

9. McCoy CE, Menchine M, Sampson S, Anderson C, Kahn C. Emergency medical services out-of-hospital scene and transport times and their association with mortality in trauma patients presenting to an urban level I trauma center. Ann Emerg Med. 2013;61(2):167-74.

10. Praça WR, Matos MCB, Fioravanti RK, Magro MCS, Hermann PRS. Perfil epidemiológico e clínico de vítimas de trauma em um hospital do Distrito Federal, Brasil. Rev Pre Infec e Saúde [Internet]. 2017;3(1):1-7. Available from: http://www.ojs.ufpi. br/index.php/nupcis/article/view/6219

Recebido em: 05/08/2018

Aceito para publicação em: 09/09/2018

Conflito de interesse: nenhum.

Fonte de financiamento: nenhuma.

\section{Endereço para correspondência:}

Phillipe Geraldo Teixeira de Abreu Reis

E-mail: phillipeareis@gmail.com adonisnasr@gmail.com

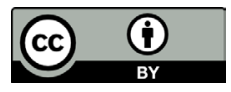

